\title{
Charting the course across the blood-brain barrier
}

\author{
David Nathanson and Paul S. Mischel
}

\author{
Department of Pathology and Laboratory Medicine and Department of Molecular and Medical Pharmacology, \\ David Geffen School of Medicine, UCLA, Los Angeles, California, USA.
}

\begin{abstract}
The blood-brain barrier (BBB) presents a significant obstacle to delivery of targeted therapies to brain tumors. In this issue of the JCI, Staquicini and colleagues apply an in vivo phage-displayed library of random peptides to identify differentially expressed peptides that can be used to transport targeted agents across the intact $\mathrm{BBB}$. The authors uncover a non-canonical, peptide-mediated iron-mimicry mechanism to induce transport of the transferrin/transferrin receptor complex across the BBB. They then demonstrate the ability of phage-targeting approaches to deliver therapeutic cargo and molecular imaging reporters across the $\mathrm{BBB}$ in an intracranial glioblastoma mouse model.
\end{abstract}

Malignant gliomas are the most common primary brain tumor in adults and one of the most lethal of all cancers. With a median survival rate of one year, they present an almost unparalleled clinical challenge. Whether they arise from the constituent cells of the brain or metastasize from other sites, gliomas are often located in the most functionally important areas of the brain, making complete surgical resection a virtual impossibility. Furthermore, most brain tumors are relatively radio- and chemoresistant, while the surrounding normal brain tissue is relatively sensitive, making attempts at successful treatment resemble Ulysses' frightening effort to pass between Scylla and Charybdis, as described in Homer's Odyssey.

Comprehensive genomic surveys have identified a number of potentially targetable mutations in glioblastoma (GBM), the most common and malignant type of glioma. These mutations, which cluster along the EGFR/PTEN/PI3K, p53, and pRb1 signaling pathways $(1,2)$, have been demonstrated to play a causative role in malignant glioma formation and progression in mouse genetic models $(3,4)$, which suggests that small-molecule inhibitors and antibodies targeting these pathways may play an important role in reshaping the future treatment of patients with GBM. However, delivering these treatments to the brain remains a significant challenge.

Conflict of interest: The authors have declared that no conflict of interest exists.

Citation for this article: J Clin Invest. 2011; 121(1):31-33. doi:10.1172/JCI45758.
The BBB: an obstacle to the delivery of brain tumor treatments

The brain is a privileged site, sheltered from the systemic circulation by the blood-brain barrier (BBB) - a structure composed of endothelial cells, associated astrocytic end-feet processes, perivascular neurons, and pericytes (Figure 1). The endothelial cells are connected by tight junctions that form an almost impenetrable barrier to all compounds except highly lipidized small molecules of less than $400 \mathrm{Da}$. Thus, delivery of the vast majority of therapeutic small molecules to the brain parenchyma is greatly limited. Brain tumors such as GBM may partially disrupt the BBB by inducing large gaps between endothelial cells (5). However, the extent of BBB disruption among individual patients, and/or among various regions within a single tumor, appears to be highly variable. Therefore, the BBB presents a significant obstacle to delivery of targeted brain tumor treatments. Attempts to artificially disrupt the BBB by intra-arterial infusion of hyperosmotic solutions presents one potential therapeutic option, as recently demonstrated by intra-arterial infusion of the VEGFspecific antibody bevacizumab after BBB disruption by mannitol in patients with recurrent malignant glioma (6). However, disruption of the BBB could potentially lead to other serious complications, such as brain edema. Therefore, development of strategies to deliver targeted agents across the $\mathrm{BBB}$ is a critical priority.

Several strategies to get therapeutic molecules across the BBB have recently been studied in malignant glioma patients. These include: (a) transnasal delivery (7); (b) convection-enhanced delivery (CED), whereby a therapeutic agent is continuously infused into the tumor bed under positive pressure to create a pressure gradient, enabling delivery to a larger region of the brain than can be achieved by diffusion (8); (c) packaging drugs and/or interfering RNAs into polyethylene glycolencapsulated liposomes that more readily cross the BBB and show tumor reduction and increase in survival in mice compared with systemic delivery, an approach that can be combined with CED $(9,10)$; (d) use of replication-competent retroviruses to deliver oncolytic therapies (11); and (e) use of mesenchymal (12) or neural stem cells to deliver small molecules, antibodies, or toxic payloads $(13,14)$. Alternatively, other investigators have sought to take advantage of endogenous BBB transporters to increase delivery of targeted agents. There are three main classes of BBB transporters: (a) carrier-mediated transporters, including the glucose and amino acid transporters; (b) active efflux transporters, including $\mathrm{P}$-glycoprotein and the other ABC gene family members; and (c) receptor-mediated transporters, of which transferrin receptor (TfR), insulin receptor, and low-density lipoprotein receptor are the best characterized (15). Attempts to target GBM by delivering EGFR-specific shRNA in PEGylated liposomes bearing insulin receptor- and TfR-specific antibodies in an in vivo model has shown some promise (16). However, these antibody-based approaches have yet to translate into the clinic. Developing the right targeting antibodies to facilitate crossing of the BBB in humans, and uncovering the molecular mechanism by which the process works, remain significant impediments to clinical application.

\section{Phage display to identify differentially expressed peptides that can be used to transport targeted agents across the intact $B B B$}

In this issue of the JCI, Staquicini et al. provide an ingenious alternative strategy to overcoming the obstacle of delivering targeted agents across the BBB, based on 


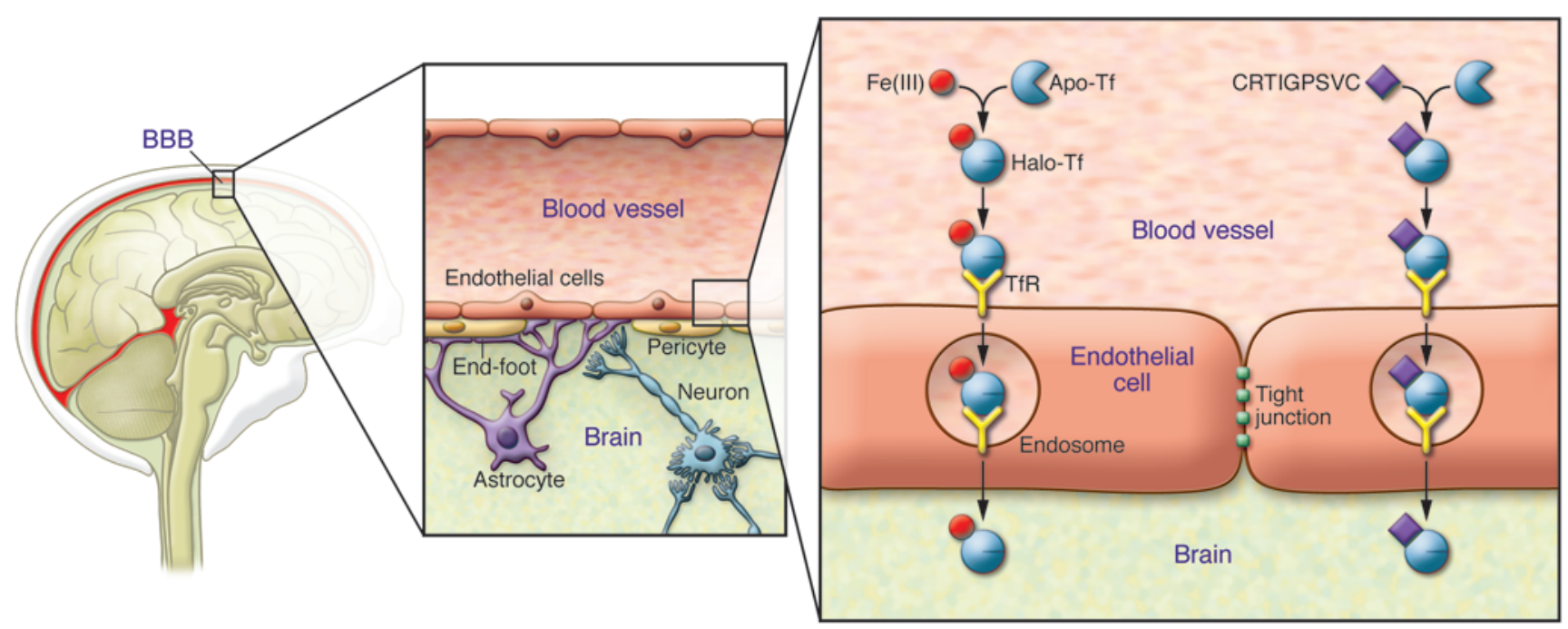

Figure 1

Peptide-mediated iron mimicry transports the transferrin/TfR complex across the BBB, delivering therapeutic and imaging cargo. The BBB is composed of a variety of cells, including endothelial cells with tight junctions, pericytes, perivascular neurons, and astrocytes. Under normal conditions, the BBB prevents the passage of small molecules into the brain. Receptor-mediated transport allows passage of selected molecules across the BBB. The binding of iron to transferrin induces a conformational shift from the open form of transferrin (Apo-Tf) to the closed form (Halo-Tf), facilitating transport of bound iron-transferrin in complex with TfR across the BBB. The iron peptide mimic CRTIGPSVC specifically binds Tf, enabling transport of the peptide and its cargo in complex with transferrin/TfR across the BBB.

identification of small peptide ligands that can be used to leverage the TfR system (17). Phage display peptide library screening, a strategy originally conceived by Smith in 1985 (18), has proven invaluable for uncovering a myriad of protein and peptide interactions. Individual bacteriophages can be engineered to express unique peptides in their phage coats, enabling screening of large random libraries against purified proteins, antibodies, or cultured cells. The bound phage particles can be recovered and each peptide ligand identified by DNA sequencing, providing a powerful, unbiased combinatorial approach to target discovery. Of importance for this application, phage display facilitates unbiased identification of receptors in their native conformation. The recovered peptides often maintain the identical biological features of the native full-length protein ligands, including biological activity (19). Therefore, the approach appears to be tailor-made for discovering functional small peptide ligands that target TfR and could therefore potentially be used to deliver targeted therapies to the brain; it might also help elucidate the mechanisms by which they work.

Pasqualini and Arap, the senior authors of the study (17), have previously used in vitro phage display to identify peptide ligands that interact with receptors on the surface by screening 60 human-derived cancer cell lines (NCI-60), revealing a series of potentially druggable targets (20). They further extended the utility of phage display as a tool for discovering drug targets by applying it to in vivo mouse models and human subjects, in the process identifying peptides that differentially interact with protein targets in vascular beds of multiple organs (including the brain) and with connective tissues such as fat, muscle, and skin, including under pathological contexts $(19,21)$. Taken together, this body of work (19-21) provides an important context for the study in this issue (17).

\section{An iron-mimicry mechanism induces transport of the transferrin/TfR complex across the BBB}

Staquicini et al. applied a phage-displayed library of random peptides to serially enrich for peptide motifs that selectively bound to endothelial cells in the brain of normal mice, in order to identify differentially expressed peptides that could be used to internalize and transport targeted agents across the intact BBB (17). Using bioinformatic strategies to identify a series of potential peptide motifs of interest, the authors focused on a particular one, CRTIGPSVC, which resides in the amino terminus of transferrin. They went on to demonstrate that CRTIGPSVCexpressing phage particles effectively cross the intact $\mathrm{BBB}$ in normal mice. Because TfR normally carries iron atoms bound to transferrin across the BBB, the authors tested the hypothesis that the peptide promotes transferrin/TfR-mediated transport by acting as an iron mimic. They uncovered an allosteric, non-canonical mechanism by which the CRTIGPSVCexpressing phage functionally mimics iron binding to the open conformation of transferrin, inducing a conformational shift from open to closed that leads to transport. This observation, in and of itself, demonstrates the benefit of phage display approaches for uncovering molecular mechanisms, since the peptides seem to retain key biological function.

The authors ground the study in clinical relevance by showing substantial overexpression of TfR in clinical GBM samples and convincingly demonstrating that the CTIGPSVC-expressing phage selectively homes to intratumoral vasculature in a well-established intracranial GBM model (17). By engineering the CRTIGPSVC peptide sequence into an adeno-associated chimeric viral vector carrying the gene encoding HSV thymidine kinase, which triggered cell suicide when the mice were treated with ganciclovir and served as a 
reporter for molecular imaging of tracers such as ${ }^{18} \mathrm{~F}$-FEAU, Staquicini et al. provide an elegant demonstration of the translational potential of phage targeting to deliver therapeutic cargos. Importantly, massive reduction of tumor size, with marked tumor cell death, was observed upon ganciclovir treatment, and noninvasive molecular imaging of these tumors made possible diagnosis and therapeutic monitoring.

\section{Concluding remarks}

It remains to be determined whether the concentration of phage-directed cargo will be therapeutically effective in tumors in patients with a largely intact BBB. However, the work of Staquicini et al. (17) provides three critical lessons. First, the work suggests the potential value of in vivo phage display for uncovering receptors that could be new drug targets through identification of ligand peptides, leveraging the power of unbiased combinatorial screening. Second, the study suggests that the specific peptide identified here, the transferrin/TfR complex-targeting peptide CRTIGPSVC, may have important therapeutic implications for delivering targeted brain tumor therapies and/or providing a platform for noninvasive imaging. Third, and perhaps most important, the study also highlights the value of this approach in uncovering potentially important functional interactions that can be leveraged using an array of possible targeting strategies. We can look forward to future applications of this approach for the discovery of new druggable targets and the identification of cell surface molecules that may be broadly important in many types of cancer as well as other diseases.

\section{Acknowledgments}

This work was supported by National Cancer Institute grant CA15189, by the Ben and Catherine Ivy Foundation, and by UCLA Tumor Biology Program United States Health and Human Services Ruth L. Kirschstein Institutional National Research Service Award T32 CA009056 (to D. Nathanson).

Address correspondence to: Paul S. Mischel, David Geffen School of Medicine, 10833 Le Conte Avenue, Los Angeles, California 90095-1732, USA. Phone: 310.794.5223; Fax: 310.206.8290; E-mail: pmischel@mednet.ucla.edu.

1. TCGA Consortium. Comprehensive genomic characterization defines human glioblastoma genes and core pathways. Nature. 2008;455(7216):1061-1068.

2. Parsons DW, et al. An integrated genomic analysis of human glioblastoma multiforme. Science. 2008; 321(5897):1807-1812.

3. Huse JT, Holland EC. Genetically engineered mouse models of brain cancer and the promise of preclinical testing. Brain Pathol. 2009;19(1):132-143.

4. Cheng CK, Fan QW, Weiss WA. PI3K signaling in glioma-animal models and therapeutic challenges. Brain Pathol. 2009;19(1):112-120.

5. Coomber BL, Stewart PA, Hayakawa K, Farrell CL, Del Maestro RF. Quantitative morphology of human glioblastoma multiforme microvessels: structural basis of blood-brain barrier defect. J New rooncol. 1985;5(4):299-307.

6. Boockvar JA, et al. Safety and maximum tolerated dose of superselective intraarterial cerebral infusion of bevacizumab after osmotic blood-brain barrier disruption for recurrent malignant glioma [published online ahead of print October 22, 2010]. J Neurosurg. doi: 10.3171/2010.9.JNS101223.

7. Tomotaka S, Toshiyasu S, Shinji Y, Hitoshi S, Yoshiharu T, Shobu S. Transnasal delivery of anticancer drugs to the brain tumor:a new strat- egy for brain tumor chemotherapy. Drug Deliv Syst. 1999;14(5):365-371.

8. Fiandaca MS, Forsayeth JR, Dickinson PJ, Bankiewicz KS. Image-guided convection enhanced delivery platform in the treatment of neurological diseases. Neurotherapeutics. 2008;5(1):123-127.

9. Bidros DS, Vogelbaum MA. Novel drug delivery strategies in neuro-oncology. Neurotherapeutics. 2009;6(3):539-546.

10. Yamashita Y, et al. Convection-enhanced delivery of a topoisomerase I inhibitor (nanoliposomal topotecan) and a topoisomerase II inhibitor (pegylated liposomal doxorubicin) in intracranial brain tumor xenografts. Neuro Oncol. 2007;9(1): 20-28.

11. Tai C, Kasahara N. Replication-competent retrovirus vectors for cancer gene therapy. Front Biosci. 2008; 13:3083-3095

12. Yong R, et al. Human bone marrow-derived mesenchymal stem cells for intravascular delivery of oncolytic adenovirus Delta24-RGD to human gliomas. Cancer Res. 2009;69(23):8932-8940.

13. Aboody KS, et al. Neural stem cells display extensive tropism for pathology in adult brain: Evidence from intracranial gliomas. Proc Natl Acad Sci U S A. 2000;97(23):12846-12851.

14. Frank RT, et al. Neural stem cells as a novel platform for tumor-specific delivery of therapeutic antibodies. PLoS ONE. 2009;4(12):e8314.

15. Pardridge WM. Blood-brain barrier delivery. Drug Discov Today. 2007;12(1-2):54-61.

16. Zhang Y, Zhang YF, Bryant J, Charles A, Boado RJ, Pardridge WM. Intravenous RNA interference gene therapy targeting the human epidermal growth factor receptor prolongs survival in intracranial brain cancer. Clin Cancer Res. 2004;10(11):3667-3677.

17. Staquicini FI, et al. Systemic combinatorial peptide selection yields a non-canonical iron-mimicry mechanism for targeting tumors in a mouse model of human glioblastoma. J Clin Invest. 2011; 121(1):161-173.

18. Smith GP. Filamentous fusion phage: novel expression vectors that display cloned antigens on the virion surface. Science. 1985;228(4705):1315-1317.

19. Staquicini FI, Sidman RL, Arap W, Pasqualini R. Phage display technology for stem cell delivery and systemic therapy. Adv Drug Deliv Rev. 2010;62(12):1213-1216.

20. Kolonin MG, et al. Ligand-directed surface profiling of human cancer cells with combinatorial peptide libraries. Cancer Res. 2006;66(1):34-40.

21. Arap W, et al. Steps toward mapping the human vasculature by phage display. Nature Med. 2002; $8(2): 121-127$ 